\title{
Pure animal phobia is more specific than other specific phobias: epidemiological evidence from the Zurich Study, the ZInEP and the PsyCoLaus
}

\author{
Vladeta Ajdacic-Gross ${ }^{1,2} \cdot$ Stephanie Rodgers ${ }^{1,2} \cdot$ Mario Müller $^{1,2}$. \\ Michael P. Hengartner, ${ }^{2,3}$. Aleksandra Aleksandrowicz ${ }^{1,2}$ - Wolfram Kawohl ${ }^{1,2}$. \\ Karsten Heekeren $^{1,2}$ - Wulf Rössler ${ }^{2,4,5} \cdot$ Jules Angst $^{1} \cdot$ Enrique Castelao $^{6}$ • \\ Caroline Vandeleur $^{6} \cdot$ Martin Preisig $^{6}$
}

Received: 24 October 2015 / Accepted: 4 March 2016 / Published online: 21 March 2016

(C) Springer-Verlag Berlin Heidelberg 2016

\begin{abstract}
Interest in subtypes of mental disorders is growing in parallel with continuing research progress in psychiatry. The aim of this study was to examine pure animal phobia in contrast to other specific phobias and a mixed subtype. Data from three representative Swiss community samples were analysed: PsyCoLaus $(n=3720)$, the ZInEP Epidemiology Survey $(n=1500)$ and the Zurich Study $(n=591)$. Pure animal phobia and mixed animal/ other specific phobias consistently displayed a low age at onset of first symptoms (8-12 years) and clear preponderance of females (OR > 3). Meanwhile, other specific phobias started up to 10 years later and displayed almost a balanced sex ratio. Pure animal phobia showed no associations with any included risk factors and comorbid disorders, in contrast to numerous associations found in the mixed subtype and in other specific phobias. Across the whole range of epidemiological parameters examined in three different samples, pure animal phobia seems to represent a different
\end{abstract}

Vladeta Ajdacic-Gross

vajdacic@dgsp.uzh.ch

1 Department of Psychiatry, Psychotherapy and Psychosomatics, Psychiatric Hospital, University of Zurich, PO Box 1930, 8021 Zurich, Switzerland

2 ZInEP, The Zurich Program for Sustainable Development of Mental Health Services, University of Zurich, Zurich, Switzerland

3 Department of Applied Psychology, Zurich University of Applied Sciences (ZHAW), Zurich, Switzerland

4 Collegium Helveticum, University of Zurich and Swiss Federal Institute of Technology, Zurich, Switzerland

5 Laboratory of Neuroscience (LIM27), Institute of Psychiatry, University of Sao Paulo, Sao Paulo, Brazil

6 Department of Psychiatry, CHUV, Lausanne, Switzerland entity compared to other specific phobias. The etiopathogenetic mechanisms and risk factors associated with pure animal phobias appear less clear than ever.

Keywords Specific phobia - Animal phobia . Epidemiology $\cdot$ Risk factors · Comorbidity $\cdot$ Methodology

\section{Introduction}

Continuing research progress is typically characterized by differentiation processes. In psychopathology, it includes both a move towards dimensional models of mental illness [37] and a growing interest on subtypes of mental disorders. Since these complementary processes still are in the early stages, their preconditions and implications remain to be considered in more detail. Their better assessment is a precondition of preparing future diagnostic systems such as anticipated by the Research Domain Criteria project of the National Institute of Mental Health [14].

The general aim of differentiating diagnoses and subtypes is to derive homogeneous entities sharing similar symptoms, similar etiopathogenetic characteristics and a similar therapeutic response. Subtyping is a two-stage process. The first stage of subtyping relies on essential criteria such as symptom clusters, alternatively or additionally also age at onset, sociodemographic characteristics, severity, course (e.g. progressive/relapsing-remitting/single episode), comorbidity patterns, risk factors, etiopathogenetic mechanisms (if known) and therapeutic success. The second stage of subtyping is less obvious to most scholars but is not less important: the operational definition of subtypes determines among other things their formal delimitations. Basically, there are two competing approaches. The subtypes can either be defined as exclusive subtypes (e.g. in 
unipolar and bipolar affective disorders) or as overlapping subtypes (e.g. in specific phobias). In exclusive subtyping, a person can be categorized in only one way, whereas in overlapping subtyping, a person can appear under two or several subtypes at the same time. An intermediate solution is found in attention deficit hyperactivity disorder: exclusive subtypes with prevailing main characteristics. The coexistence of different operational definitions in the Diagnostic and Statistical Manual (DSM) and in the International Classification of Diseases (ICD) is indicative of lacking awareness of this prerequisite.

It is noteworthy that the choice of operational definitions can have consequences that are as far-reaching as those associated with the essential features of subtypes. Obsessive-compulsive disorder provides an informative example [34]. In general, the larger the differences between subtypes of a disorder, the greater the consequences of choosing between alternative operational definitions. In this study, we illustrate such consequences in specific phobia (SP).

SP is one of the most prevalent mental disorders with lifetime prevalence rates of $10 \%$ [6] to $35 \%$ [7]. A large European study yielded an "expert-based best estimate" of the 12-month prevalence rate of $6.4 \%$ [38]. SP symptoms generate moderate distress levels compared to symptoms in other common mental disorders (CMD) [1]. Well-known epidemiological features of SP include a sex ratio of about 2 , an early age at onset, mostly in childhood, and moderate comorbidity rates compared to other CMD [6]. However, relatively few risk factors have been evaluated and confirmed up to now [35].

The DSM-IV and DSM-5 consider five subtypes of SP: animal, natural environment, blood-injection-injury, situational and other. Both epidemiological [6, 29-31] and neuroimaging $[16,25]$ evidence suggests more or less substantial differences between animal, environmental and other specific phobias. Here, we focused on animal phobia, a common and well-characterized subtype of SP, and compared it to the rest of the SPs. We implemented the conventional operational definition of SP subtypes (animal phobias, other SPs), which allows for overlapping categories, and contrasted it with the alternative operational definition of exclusive SP subtypes (pure animal phobias, other SPs, mixed animal and other SPs). In the first instance, subjects with SP were divided into two (optionally overlapping) categories, and in the second instance into three (necessarily exclusive) categories. We examined the outcomes with regard to the most relevant epidemiological parameters, risk factors and comorbidity patterns. Thus, not specific hypotheses but shifts and adjustments in association patterns depending on the operational definitions of SP subtypes were in the focus of this study. We replicated the analyses in three Swiss data sets, i.e. PsyCoLaus, the ZInEP Epidemiology Survey and the Zurich Study.

\section{Materials and methods}

\section{The PsyCoLaus sample}

The PsyCoLaus study [33] is the psychiatric part of the population-based CoLaus study [20]. Participants in CoLaus $(n=6736)$ were randomly selected between 2003 and 2006 in the city of Lausanne (Switzerland) based on the population register of the city. They were assessed at an outpatient clinic [20,27]. The study protocol consisted of collecting clinical data and blood samples, as well as conducting an interview with a semi-structured questionnaire [27]. One year later, all participants in CoLaus aged $35-66$ years $(n=5535)$ were invited to participate in PsyCoLaus; a total of 3720 individuals $(67 \%)$ took part (see Table 1) [33]. A major aim of the PsyCoLaus study was to record data on the prevalence of threshold and subthreshold psychiatric syndromes.

The psychiatric part of the assessment within the PsyCoLaus study included the French version of the semi-structured Diagnostic Interview for Genetic Studies (DIGS) [28, 32]. The DIGS collects information on a broad spectrum of DSM-IV Axis I criteria and, moreover, on the course and chronology of comorbid features [33]. The brief phobia section of the DIGS was replaced by the corresponding but more extensive sections of the Schedule for Affective Disorders and Schizophrenia-Lifetime and Anxiety disorder version (SADS-LA) [19], which elicited detailed information relating to the DSM-IV criteria for agoraphobia with or without panic attacks, social phobias and SPs. The DIGS and the SADS-LA figures represent lifetime diagnoses of CMD. Inter-rater and test-retest reliability of the French version of the DIGS was successfully established for major
Table 1 Characteristics of PsyCoLaus, the ZInEP epidemiology survey and the Zurich Study

\begin{tabular}{llll}
\hline & PsyCoLaus & ZInEP & Zurich Study \\
\hline Year of interview(s) & $2004-2007$ & $2010-2012$ & $1979,1981,1986,1988,1993,1999,2008$ \\
$N$ screening & - & 9829 & 4547 \\
$N$ sample & 3720 & 1500 & 591 \\
$N$ after offsetting stratification & - & 3600 & 2600 \\
Age range & $35-66$ & $20-41$ & $20-50$ \\
\hline
\end{tabular}


mood and psychotic disorders [32] as well as for substance use and antisocial personality disorders [8]. Inter-rater and test-retest reliability was also successfully established for the anxiety sections of the French version of the SADS-LA [24].

\section{The ZInEP epidemiology sample}

The ZInEP epidemiology survey [2] is a subproject of the Zurich Program for Sustainable Development of Mental Health Services (ZInEP; German: Zürcher Impulsprogramm zur nachhaltigen Entwicklung der Psychiatrie), which was designed in order to enhance the development of psychiatry in the canton of Zurich (Switzerland). The epidemiology survey consists of four components: (1) a brief telephone screening, (2) a comprehensive semi-structured interview supplemented by self-report questionnaires, (3) a series of socio- and neurophysiological tests focusing on stress and psychotic symptoms and (4) a longitudinal survey. The survey was carried out between August 2010 and September 2012. For the current article, data from the first two study parts were used, which are methodologically adapted from the Zurich Study.

First, 9829 subjects representative of the canton of Zurich were screened by a computer-assisted telephone interview (CATI) using the SCL-27 [21]. The records of the screening sample were randomly chosen from the communal public authority register. The sample was restricted to 20- to 41-year-old adults with Swiss nationality. In cases where the person in question could be reached by telephone, the response rate was $73.9 \%$. The overall response rate was $53.6 \%$.

After the screening procedure, 1500 participants from this initial screening sample were selected at random and interviewed in detail by comprehensive face-to-face interview. Of those subjects invited to an interview appointment, $64.9 \%$ actually showed up. A stratified sample procedure was applied that included $60 \%$ high-scorers and $40 \%$ low-scorers scorers (cut-off criterion: 75th percentile of the GSI of the SCL-27 [21]) using a shortened version of the SPIKE (Structured Psychopathological Interview and Rating of the Social Consequences of Psychological Disturbances for Epidemiology) [4]. Diagnoses of CMD were computed as 12-month prevalence rates according to DSMIV criteria. Exceptions included:

- diagnosis of generalized anxiety disorder (GAD) with a modified time criterion of one month;

- diagnosis of agoraphobia, where the criteria were modified according to DSM-V (number of symptoms, coding of impairment), since the DSM-IV criteria of agoraphobia diverged from the common diagnosis scheme;
- diagnosis of neurasthenia, which was based on ICD-10 criteria;

- diagnosis of mania/hypomania and bipolar disorders, which were adapted to the criteria of the Bridge Study [3].

The technical details of the ZInEP epidemiology survey have been published in Ajdacic-Gross et al. [2].

\section{The Zurich Study sample}

The prospective longitudinal Zurich Study [4, 5] comprises a sample of 591 subjects interviewed at seven time points from 1979 to 2008 . This sample was composed of 299 females and 292 males (born in 1958 and 1959, respectively). Based on the 85th percentile of the Global Severity Index (GSI) of the SCL-90-R [17], the sample was stratified in two-thirds of randomly selected high scorers and one-third of randomly drawn low scorers. Of the original sample, 335 subjects $(57 \%)$ participated in the last follow-up in 2008. The detailed participation rates were: $43 \%$ in all seven interviews; $13 \%$ in six interviews; $11 \%$ in five interviews; $9 \%$ in four interviews; $7 \%$ in three interviews; $9 \%$ in two interviews; and $9 \%$ in one interview.

The main instrument in the Zurich Study was the Structured Psychopathological Interview and Rating of Social Consequences of Psychic Disturbances for Epidemiology (SPIKE) [4]. It enables psychiatric diagnoses according to the respective version of the DSM criteria available at the time of interview. The CMD rates are based on aggregated information from up to seven interviews. In each interview, the diagnoses were assessed for the last 12-month period. The inter-rater reliability and validity of the SPIKE have been successfully confirmed $[22,26]$.

\section{Interviewers}

In all three studies, the interviews were carried out by trained psychologists or psychiatrists. Supervision was provided both by the scientific study staff and by senior interviewers.

\section{Approval by ethics committees}

All studies are in strict accordance with the Declaration of Helsinki of the World Medical Association. All studies were approved by the ethics committees of either the university (PsyCoLaus [33]) or local/cantonal committees (ZInEP, Zurich Study) concerned. All participants gave their written informed consent. 


\section{Weighting}

The Zurich Study and the ZInEP sample are both stratified samples. Stratification is a common epidemiological strategy used either to oversample a smaller subgroup or, as in this case, to save resources [18]. If the stratification were offset, the Zurich Study would represent 2600 subjects and the ZInEP Epidemiology Survey 3600 subjects. The corresponding design weights are 11.3 in the former and 4.5 in the latter study. In the ZInEP Epidemiology Survey, additional weights were introduced in order to account for nonresponse/under-representation according to urbanicity status, education level and the weight of a singular sex-birth year subgroup within the population of the 20- to 40-yearold subjects. Statistical analysis of stratified samples requires modelling procedures which take the strata and the weights into account (see "Statistical analysis" below).

\section{Sociodemographic variables}

We included the basic sociodemographic variables sex, age and education level. Education level was assessed as a tripartite variable (low, medium and high). Since the outcome of this variable depends on the age of the interviewee, the comparability between the studies is limited. In the Zurich Study, we used information gathered at age 20 and differentiated between basic school level, apprenticeship level and university-track education. In ZInEP and in PsyCoLaus, basic school and apprenticeship level were classified as "low" category, pre-university and high-level technical schools as "medium" category and university level as "high" category.

\section{Familial aggregation}

The familial aggregation of mental and substance use problems was assessed in PsyCoLaus by implementing the whole family, whereas in ZInEP and in the Zurich Study the data related to first-degree relatives.

\section{Childhood adversities}

Discrepancies between the studies are also found in respect of adversities in childhood and traumatic experiences across the lifespan. Childhood adversities were assessed by lists in all questionnaires. In ZInEP, we additionally introduced the Childhood Trauma Questionnaire [9].

\section{Statistical analysis}

In the statistical analysis, we implemented basic descriptive models, cross-tabulations and regression models. Programming and most analyses were carried out with SPSS.
Adjustment according to the sample stratification was necessary in ZInEP and in Zurich Study, and was performed by the SAS survey procedures SURVEYFREQ, SURVEYMEANS and SURVEYLOGISTIC. The results were presented together with confidence intervals and also specific formatting in order to assign at the same time conventional significance levels (.01 and .05). No corrections for multiple testing were applied.

\section{Results}

The outcomes of univariate and bivariate analyses related to sociodemographic variables and risk factors in pure subtypes are displayed in Tables 2, 4 and 6.

The overall prevalence rates of SPs ranged between $12.1 \%$ (ZInEP-12-month rates) and $21.4 \%$ (Zurich Study-aggregated rates across 30 years/seven interviews). The figures mostly varied in the mixed subtype (2.9-8.3\%) and somewhat less in the pure animal subtype (3.2-5.9\%), whereas the other SPs subtype showed congruent rates (range 6.0-7.2\%) over all three studies.

The SPs were consistently more frequent in females than in males, however again strongly varying by subtype. In pure animal phobia and the mixed subtype, the sex ratio reached a value of three or above in all studies. It was consistently highest in the mixed subtype. In other SPs, it ranged between 1.1 and 1.5, not necessarily achieving a common statistical significance level. Similarly, the age at onset diverged between pure animal phobias (range 7.710.1) versus other SPs (range 15.0-18.7), with the mixed type being closer to the former. The results clearly diverged less if conventional overlapping SP subtypes were analysed instead of exclusive subtypes.

Education level as an indicator of socioeconomic status (SES) of the participant's family showed consistent patterns only with respect to the mixed subtype. However, the effects were moderate and achieved common significance levels only in the PsyCoLaus study.

The results related to risk factors and comorbidity patterns were shaped by the methodological differences between the three studies. Nevertheless, the tables indicate that these associations concentrated in other SPs and the mixed subtype. Relevant associations appeared to arise only sporadically in pure animal phobias.

Familial aggregation of CMD involved typically phobias and anxiety, but also OCD and affective disorders. The mixed subtype attracted more and stronger associations than pure animal phobias and other SPs, and also included positive associations with substance abuse (ZInEP).

Traumatic experiences in childhood assessed by the Childhood Trauma Questionnaire yielded prominent associations with other SPs particularly in ZInEP. Other 
Table 2 Sociodemographic marker variables and risk factors related to specific phobias (lifetime diagnosis) in PsyCoLaus and calculations based on lifetime diagnoses

\begin{tabular}{|c|c|c|c|c|c|c|}
\hline & \multicolumn{3}{|c|}{ Exclusive subtypes } & \multicolumn{2}{|c|}{ Overlapping subtypes } & \multirow[t]{2}{*}{ Overall } \\
\hline & Pure animal & Mixed & Pure other & Animal & Other & \\
\hline \multicolumn{7}{|l|}{ Prevalence } \\
\hline$n($ rate in $\%)$ & $129(3.5)$ & $181(4.9)$ & $271(7.3)$ & $310(8.3)$ & $452(12.2)$ & $581(15.6)$ \\
\hline \multicolumn{7}{|l|}{ Sex } \\
\hline$n$ (rate in \%) males & $27(1.5)$ & $35(2.0)$ & $113(6.5)$ & $62(3.5)$ & $148(8.5)$ & $175(10.1)$ \\
\hline$n$ (rate in \%) females & $102(5.2)$ & $146(7.4)$ & $158(8.0)$ & 248 (12.6) & $304(15.4)$ & $406(20.7)$ \\
\hline OR females:males (95\% CI) & $3.5(2.3-5.4)$ & $3.9(2.7-5.7)$ & $1.3(1.0-1.6)$ & $3.9(2.9-5.2)$ & $2.0(1.6-2.4)$ & $2.3(1.9-2.8)$ \\
\hline Age at onset: mean $(95 \% \mathrm{CI})$ & $8.3(7.1-9.4)$ & $12.0(10.4-13.5)$ & $18.3(16.6-19.9)$ & $10.4(9.4-11.5)$ & $15.7(14.5-16.9)$ & $14.1(13.1-15.1)$ \\
\hline \multicolumn{7}{|l|}{ Education: $n$ (rate) } \\
\hline Low & $71(3.6)$ & $114(5.8)$ & $151(7.7)$ & $185(9.4)$ & $265(13.5)$ & $336(17.2)$ \\
\hline Medium & $34(3.7)$ & $34(3.7)$ & $61(6.7)$ & $68(7.4)$ & $95(10.4)$ & $129(14.1)$ \\
\hline University & $24(3.0)$ & $29(3.6)$ & $55(6.9)$ & $53(6.6)$ & $84(10.5)$ & $108(13.6)$ \\
\hline \multicolumn{7}{|c|}{ Familial aggregation (odds ratios $95 \% \mathrm{CI}$ ) } \\
\hline Depression (parents) & $1.1(0.7-1.7)$ & $1.4(1.0-2.1)$ & $1.3(1.0-1.8)$ & $1.3(1.0-1.7)$ & $1.4(1.1-1.8)$ & $1.3(1.1-1.7)$ \\
\hline Depression (family) & $1.4(1.0-2.1)$ & $1.1(0.7-1.5)$ & $1.3(1.0-1.7)$ & $1.2(0.9-1.6)$ & $1.2(0.9-1.5)$ & $1.3(1.0-1.6)$ \\
\hline Anxiety (family) & $1.4(0.8-2.4)$ & $1.0(0.6-1.7)$ & $1.8(1.3-2.6)$ & $1.2(0.8-1.8)$ & $1.5(1.1-2.1)$ & $1.6(1.1-2.1)$ \\
\hline Bipolar disorder (family) & $0.8(0.2-2.5)$ & $0.6(0.2-1.8)$ & $0.8(0.3-1.7)$ & $0.6(0.3-1.5)$ & $0.7(0.3-1.3)$ & $0.7(0.4-1.2)$ \\
\hline OCD (family) & * & $1.8(0.5-5.9)$ & $0.8(0.2-3.2)$ & $1.0(0.3-3.3)$ & $1.2(0.5-3.0)$ & $0.9(0.3-2.2)$ \\
\hline Schizophrenia (family) & $1.6(0.5-5.2)$ & $0.7(0.2-3.1)$ & $1.9(0.8-4.2)$ & $1.1(0.4-2.8)$ & $1.4(0.7-2.9)$ & $1.5(0.8-2.9)$ \\
\hline \multicolumn{7}{|c|}{ Childhood adversity (odds ratios $95 \% \mathrm{CI}$ ) } \\
\hline Parents beating each other & $0.7(0.4-1.3)$ & $1.5(1.0-2.1)$ & $1.2(0.9-1.7)$ & $1.1(0.8-1.6)$ & $1.3(1.0-1.7)$ & $1.2(0.9-1.5)$ \\
\hline $\begin{array}{l}\text { Fear from maltreatment by } \\
\text { parents }\end{array}$ & $0.9(0.5-1.6)$ & $1.7(1.1-2.5)$ & $1.7(1.2-2.4)$ & $1.3(0.9-1.8)$ & $\mathbf{1 . 8}(1.3-2.3)$ & $1.6(1.2 .-2.1)$ \\
\hline Run away from home & $1.2(0.5-3.0)$ & $2.7(1.5-4.8)$ & $0.8(0.4-1.7)$ & $2.1(1.3-3.5)$ & $1.6(1.1-2.5)$ & $1.6(1.0-2.5)$ \\
\hline
\end{tabular}

$O R$ odds ratios, $O C D$ obsessive-compulsive disorder

* Left blank because of 0-cells or low frequencies

Significance levels: $p<.05$ (bold)

childhood adversities were either associated with the mixed subtype or with other SPs without a consistent pattern over the three studies. Furthermore, running away from home was prominently and consistently associated with the mixed subtype.

Also in the analysis of comorbidities (see Tables 3, 5, 7), pure animal phobias lacked associations in all three studies. The focus was again on the mixed subtype and other SPs, for which the associations with other anxiety disorders and OCD were most outstanding. These associations were typically stronger with respect to the mixed subtype than to other SPs.

\section{Discussion}

This study investigated the consequences of choosing different operational definitions for two large subtypes of specific phobia- animal phobias and other SPs. The analyses were replicated in three large Swiss epidemiological data sets. On the one hand, we analysed exclusive subtypes (pure animal phobias, other SPs and the mixed subtype) and, on the other hand, conventionally defined subtypes, i.e. optionally overlapping subtypes (animal, other SPs). The use of exclusive subtypes led to surprising results with fundamental implications. Across the whole range of epidemiological parameters and across all three data sets, pure animal and other phobias shared no common characteristics at all. The results suggest that pure animal and other phobias could represent two completely different entities. They appear to be more different than any average pair of common mental disorders. In the following, we will draw preliminary conclusions from the findings and in a second step discuss further general implications.

\section{Preliminary integration of the findings}

Changing the operational definition to pure subtypes in SP revealed differences between animal phobias and other SPs 
Table 3 Comorbidity patterns in specific phobias in PsyCoLaus; odds ratios and confidence intervals (95\%); and calculations based on lifetime diagnoses

\begin{tabular}{|c|c|c|c|c|c|c|}
\hline & \multicolumn{3}{|c|}{ Exclusive subtypes } & \multicolumn{2}{|c|}{ Overlapping subtypes } & \multirow[t]{2}{*}{ Overall } \\
\hline & Pure animal & Mixed & Pure other & Animal & Other & \\
\hline MDD, single & $1.0(0.7-1.5)$ & $1.1(0.8-1.5)$ & $1.1(0.8-1.4)$ & $1.0(0.8-1.4)$ & $1.1(0.9-1.4)$ & $1.1(0.9-1.3)$ \\
\hline MDD, recurrent & $1.8(1.2-2.6)$ & $2.1(1.5-2.9)$ & $1.4(1.0-1.9)$ & $2.0(1.5-2.6)$ & $1.7(1.4-2.1)$ & $1.8(1.5-2.2)$ \\
\hline MDD & $1.5(1.0-2.1)$ & $1.8(1.3-2.4)$ & $1.3(1.0-1.7)$ & $1.7(1.3-2.1)$ & $1.5(1.2-1.9)$ & $1.5(1.3-1.8)$ \\
\hline Dysthymia & $0.8(0.3-2.3)$ & $1.4(0.7-2.8)$ & $1.1(0.6-2.1)$ & $1.2(0.7-2.1)$ & $1.3(0.8-2.0)$ & $1.2(0.7-1.8)$ \\
\hline Bipolar disorder I/II & $1.3(0.4-4.3)$ & $2.4(1.1-5.3)$ & $0.8(0.3-2.3)$ & $2.0(1.0-3.9)$ & $1.5(0.8-2.8)$ & $1.6(0.9-2.9)$ \\
\hline GAD & $1.4(0.5-3.8)$ & $2.7(1.4-5.4)$ & $2.8(1.6-5.0)$ & $2.2(1.2-4.0)$ & $3.1(1.9-5.0)$ & $2.9(1.8-4.5)$ \\
\hline Agoraphobia & $0.9(0.4-2.2)$ & $6.9(4.7-10.4)$ & $2.0(1.2-3.2)$ & $4.3(2.9-6.2)$ & $4.5(3.2-6.2)$ & $3.7(2.7-5.2)$ \\
\hline Social phobia & $1.7(1.1-2.7)$ & $2.4(1.7-3.5)$ & $1.7(1.2-2.3)$ & $2.2(1.7-3.0)$ & $2.1(1.6-2.7)$ & $2.1(1.7-2.6)$ \\
\hline OCD & $1.9(0.6-6.2)$ & $4.8(2.3-10.1)$ & $1.9(0.8-4.4)$ & $3.9(2.0-7.5)$ & $3.4(1.9-6.5)$ & $3.4(1.9-6.2)$ \\
\hline Panic disorder & $0.5(0.2-1.6)$ & $4.2(2.7-6.5)$ & $2.3(1.5-3.6)$ & $2.5(1.7-3.8)$ & $3.5(2.5-4.9)$ & $2.8(2.0-3.9)$ \\
\hline Overanxious disorder & $1.6(0.9-2.8)$ & $2.7(1.8-4.2)$ & $1.4(0.9-2.2)$ & $2.3(1.6-3.3)$ & $2.1(1.5-2.8)$ & $2.0(1.5-2.8)$ \\
\hline Separation anxiety disorder & $1.8(1.0-3.3)$ & $2.7(1.7-4.3)$ & $1.3(0.8-2.2)$ & $2.4(1.7-3.6)$ & $\mathbf{2 . 0}(1.4-2.8)$ & $2.1(1.5-2.9)$ \\
\hline ADHD & $1.2(0.4-3.4)$ & $3.2(1.8-5.9)$ & $1.3(0.7-2.7)$ & $2.4(1.4-4.2)$ & $2.2(1.4-3.6)$ & $2.0(1.3-3.2)$ \\
\hline Conduct disorder & $0.8(0.2-2.5)$ & $1.3(0.6-2.9)$ & $0.6(0.2-1.5)$ & $1.1(0.6-2.1)$ & $0.9(0.5-1.6)$ & $0.9(0.5-1.5)$ \\
\hline Oppositional defiant disorder & $0.7(0.2-2.9)$ & $2.6(1.3-5.2)$ & $1.0(0.4-2.4)$ & $1.8(0.9-3.4)$ & $1.7(1.0-3.0)$ & $1.5(0.9-2.5)$ \\
\hline PTSD & $0.8(0.3-1.9)$ & $3.7(2.4-5.7)$ & $1.3(0.8-2.2)$ & $2.4(1.6-3.6)$ & $2.4(1.7-3.4)$ & $2.0(1.4-2.8)$ \\
\hline Bulimia & $0.5(0.1-3.7)$ & $1.5(0.5-4.3)$ & $1.3(0.5-3.2)$ & $1.1(0.4-2.8)$ & $1.4(0.7-2.9)$ & $1.1(0.6-2.4)$ \\
\hline Alcohol abuse/dependence & $0.7(0.4-1.3)$ & $1.0(0.6-1.5)$ & $1.1(0.8-1.6)$ & $0.8(0.6-1.2)$ & $1.1(0.8-1.4)$ & $1.0(0.7-1.3)$ \\
\hline Marijuana abuse/dependence & $1.2(0.6-2.4)$ & $0.7(0.3-1.5)$ & $1.3(0.8-2.1)$ & $0.9(0.5-1.5)$ & $1.0(0.7-1.6)$ & $1.1(0.7-1.6)$ \\
\hline Illicit drugs abuse & $1.4(0.3-6.0)$ & $1.0(0.2-4.2)$ & $1.4(0.5-3.9)$ & $1.2(0.4-3.4)$ & $1.2(0.5-3.0)$ & $1.3(0.6-2.8)$ \\
\hline
\end{tabular}

$M D D$ major depression disorder; $G A D$ generalized anxiety disorder; $O C D$ obsessive-compulsive disorder; $A D H D$ attention deficit hyperactivity disorder, $P T S D$ post-traumatic stress disorder

Significance levels: $p<.05$ (bold)

that are much greater than believed up to now. Pure animal phobias were distinctly preponderant among females and showed a particularly low age at onset. Even more important: they were marginally associated with any risk factors or further covariates-much less than hitherto indicated by studies on SP [6] — and displayed almost no comorbidities with other mental disorders.

In contrast, other specific phobias displayed neither a remarkable sex ratio nor an extreme average age at onset. Nevertheless, they were associated with common risk factors such as traumatic experiences in childhood and engaged in comorbidities mostly with other anxiety disorders. They gave the impression of a disorder that is not too different from other anxiety disorders.

In fact, research findings have up to now suggested few comorbid associations in animal phobias, for example, with depression in a reanalysis of the National Comorbidity Survey data [12] or with a series of disorders in the Dresden Mental Health Study [6] — but a broad network of associations in other SPs. Among others, environmental and situational phobias were found to be associated with social phobia [11, 15, 31], agoraphobia [15], GAD [29], separation anxiety disorder [11, 29], OCD [31] and PTSD [11]. These associations were replicated in this study both in the other SPs and in the mixed subtype.

In addition, findings from the neurobiological strand of research are in line with serious discrepancies between animal and other phobias. The HPA axis seems to not be activated in animal (spider) phobias [36]. In addition, disgust sensitivity (or disgust proneness) has been repeatedly found to be associated with SPs, in animal (spider) phobia notably to a greater extent than fear [13, 39]. A study comparing spider and blood-injection-injury phobias found that the former was associated with core disgust, i.e. the avoidance of infection traits via the oral route, whereas the latter was associated with animal reminder disgust [10]. Complementarily, the results of this study indicate that the discrepancies between pure animal phobias and other SPs are far-reaching, i.e. substantial, and not only of quantitative nature.

As a consequence, understanding the mixed subtype becomes an additional challenge. Besides selectively sharing characteristics with the pure animal phobias and other SPs, the mixed subtype stands out in two ways. First, there were more frequent and typically stronger associations with risk factors and other disorders, thus indicating a more 
Table 4 Sociodemographic marker variables and risk factors related to specific phobias in ZInEP and calculations based on 12-month diagnoses

\begin{tabular}{|c|c|c|c|c|c|c|}
\hline & \multicolumn{3}{|c|}{ Exclusive subtypes } & \multicolumn{2}{|c|}{ Overlapping subtypes } & \multirow[t]{2}{*}{ Overall } \\
\hline & Pure animal & Mixed & Pure other & Animal & Other & \\
\hline \multicolumn{7}{|l|}{ Prevalence } \\
\hline$n$ (rate in $\%$, weighted) & $53(3.2)$ & $55(2.9)$ & $112(6.0)$ & $108(6.1)$ & $167(9.0)$ & $220(12.1)$ \\
\hline \multicolumn{7}{|l|}{ Sex } \\
\hline$n$ (rate in $\%$, weighted) males & $17(1.5)$ & $14(1.0)$ & $52(5.2)$ & $31(2.5)$ & $66(6.2)$ & $83(7.7)$ \\
\hline$n$ (rate in $\%$, weighted) females & $36(4.8)$ & $41(4.9)$ & $60(6.9)$ & $77(9.7)$ & $101(118)$ & $137(16.6)$ \\
\hline $\begin{array}{l}\text { OR females:males (95\% CI) } \\
\text { (weighted) }\end{array}$ & $\mathbf{3 . 3}(1.5-7.2)$ & $5.3(2.3-12.3)$ & $1.3(0.8-2.3)$ & $4.3(2.4-7.6)$ & $2.0(2.0-3.2)$ & $2.4(1.6-3.6)$ \\
\hline Age at onset, mean weighted $(95 \% \mathrm{CI})$ & $7.7(6.3-9.1)$ & $10.1(7.0-13.2)$ & $15.0(12.8-17.3)$ & $8.7(7.2-10.2)$ & $13.1(11.5-14.8)$ & $11.5(10.2-12.8)$ \\
\hline \multicolumn{7}{|l|}{ Education: $n$ (rate in $\%$, weighted) } \\
\hline Low & $22(3.2)$ & $27(3.5)$ & $54(6.9)$ & $49(6.8)$ & $81(10.4)$ & $103(13.7)$ \\
\hline Medium & $24(3.4)$ & $19(2.4)$ & $46(5.4)$ & $43(5.7)$ & $65(7.9)$ & $89(11.1)$ \\
\hline University & $7(2.9)$ & $9(2.0)$ & $12(4.3)$ & $16(4.9)$ & $21(6.3)$ & $28(9.2)$ \\
\hline \multicolumn{7}{|c|}{ Familial aggregation $^{\text {a }}$ (weighted, ORs, $95 \%$ CI) } \\
\hline Hyperactivity & $0.6(0.2-1.6)$ & $1.4(0.6-3.4)$ & $1.2(0.6-2.5)$ & $1.0(0.5-1.9)$ & $1.3(0.7-2.3)$ & $1.1(0.6-1.9)$ \\
\hline Specific phobia & $2.7(1.3-5.7)$ & $3.6(1.7-7.9)$ & $2.1(1.2-3.7)$ & $3.3(1.9-5.7)$ & $2.7(1.7-4.2)$ & $2.9(1.9-4.3)$ \\
\hline Other anxiety disorders & $0.8(0.3-2.0)$ & $3.4(1.4-8.2)$ & $2.7(1.5-4.9)$ & $1.8(0.9-3.5)$ & $3.1(1.9-5.2)$ & $2.4(1.5-3.8)$ \\
\hline Depression & $0.9(0.4-1.8)$ & $1.9(0.9-4.4)$ & $1.6(0.9-2.8)$ & $1.3(0.6-1.7)$ & $1.8(1.1-2.8)$ & $1.5(1.0-2.2)$ \\
\hline Bipolar disorders & $2.0(0.8-5.0)$ & $1.6(0.5-6.6)$ & $0.5(0.2-1.3)$ & $2.0(0.9-4.3)$ & $0.9(0.4-2.1)$ & $0.9(0.4-2.1)$ \\
\hline OCD & $2.2(0.9-5.5)$ & $3.0(1.2-7.6)$ & $1.7(0.8-3.6)$ & $2.7(1.4-5.3)$ & $2.2(1.2-4.1)$ & $2.4(1.4-4.0)$ \\
\hline Alcohol abuse & $0.7(0.3-1.9)$ & $1.9(0.8-4.5)$ & $\mathbf{0 . 4}(0.2-0.8)$ & $1.3(0.7-2.4)$ & $0.8(0.5-1.4)$ & $0.8(0.5-1.3)$ \\
\hline Illicit drugs abuse & $1.7(0.5-5.5)$ & $2.6(0.9-7.6)$ & $1.2(0.6-2.8)$ & $2.2(1.0-5.0)$ & $1.7(0.9-3.4)$ & $1.8(0.9-3.3)$ \\
\hline \multicolumn{7}{|l|}{ CTQ subscales ${ }^{\mathrm{b}}$ (weighted, ORs, $95 \% \mathrm{CI}$ ) } \\
\hline Emotional abuse & $1.0(0.6-1.9)$ & $1.9(1.1-3.2)$ & $1.5(1.1-2.0)$ & $1.5(1.0-2.3)$ & $1.7(1.2-2.3)$ & $1.6(1.2-2.1)$ \\
\hline Emotional neglect & $0.7(0.4-1.3)$ & $1.2(0.7-2.3)$ & $1.6(1.2-2.1)$ & $1.0(0.6-1.5)$ & $1.5(1.1-2.0)$ & $1.3(1.0-1.7)$ \\
\hline Physical abuse & $1.0(0.3-3.2)$ & $1.6(0.8-3.2)$ & $1.6(0.9-2.8)$ & $1.3(0.7-2.5)$ & $1.7(1.0-2.7)$ & $1.5(0.9-2.4)$ \\
\hline Sexual abuse & $0.8(0.4-1.8)$ & $1.3(0.8-2.2)$ & $1.5(1.0-2.1)$ & $1.1(0.7-1.7)$ & $1.5(1.1-2.0)$ & $1.3(1.0-1.8)$ \\
\hline \multicolumn{7}{|c|}{ Other childhood adversity (weighted, ORs, $95 \% \mathrm{CI}$ ) } \\
\hline Parents quarrelled often & $1.3(0.6-2.8)$ & $2.2(0.9-5.7)$ & $1.2(0.6-2.2)$ & $1.7(0.9-3.3)$ & $1.5(0.9-2.5)$ & $1.5(0.9-2.4)$ \\
\hline Frequently punished or criticized & $1.0(0.4-2.4)$ & $1.5(0.6-4.2)$ & $1.5(0.8-2.7)$ & $1.3(0.6-2.5)$ & $1.5(0.9-2.6)$ & $1.4(0.9-2.2)$ \\
\hline Run away from home & $1.1(0.4-2.7)$ & $5.4(2.1-14.0)$ & $1.2(0.6-2.5)$ & $2.7(1.3-5.7)$ & $2.3(1.2-4.4)$ & $2.0(1.1-3.5)$ \\
\hline Many friends in school & $1.1(0.5-2.3)$ & $1.4(0.6-3.1)$ & $0.7(0.4-1.3)$ & $1.2(0.7-2.1)$ & $0.8(0.5-1.4)$ & $0.9(0.6-1.4)$ \\
\hline Bullied in school & $1.7(0.8-3.8)$ & $1.2(0.5-2.9)$ & $1.3(0.7-2.4)$ & $1.5(0.8-2.7)$ & $1.3(0.8-2.2)$ & $1.4(0.9-2.2)$ \\
\hline Quarrels in school & $2.3(0.9-5.4)$ & $1.0(0.3-3.4)$ & $1.4(0.6-3.5)$ & $1.6(0.8-3.4)$ & $1.3(0.6-2.7)$ & $1.6(0.9-2.9)$ \\
\hline Often expelled or disregarded & $1.2(0.5-2.6)$ & $1.0(0.4-2.5)$ & $1.2(0.6-2.4)$ & $1.1(0.6-2.0)$ & $1.1(0.6-2.0)$ & $1.2(0.7-1.9)$ \\
\hline Afraid of gym or swimming & $0.9(0.4-1.9)$ & $1.9(0.7-5.2)$ & $2.0(1.1-3.9)$ & $1.3(0.6-2.6)$ & $2.1(1.2-3.6)$ & $1.7(1.0-2.8)$ \\
\hline
\end{tabular}

$O R$ odd's ratios, $A D H D$ attention deficit hyperactivity disorder, $O C D$ obsessive-compulsive disorder

Significance levels: $p<.05$ (bold)

a Restricted to first-degree relatives

b CTQ: Childhood Trauma Questionnaire

severe subtype and, probably, more extensive involvement of different brain networks. Second, the broad range of familial aggregation (ZInEP) and family burden issues (Zurich Study) indicated a different and more serious background. Among others, this also explains associations with a variable such as "running away from home", which at first glance appear to be spurious.

\section{Limitations}

This study focuses solely on pure animal phobias. Including more than one pure subtype results in a rapidly increasing number of mixed subtypes, thus requiring either pooling of data sets or use of extra-large data sets such as the World Health Organization world mental health surveys [23]. 
Table 5 Comorbidity patterns in specific phobias in ZInEP; odds ratios and confidence intervals (95\%); and calculations based on 12-month diagnoses

\begin{tabular}{|c|c|c|c|c|c|c|}
\hline & \multicolumn{3}{|c|}{ Exclusive subtypes } & \multicolumn{2}{|c|}{ Overlapping subtypes } & \multirow[t]{2}{*}{ Overall } \\
\hline & Pure animal & Mixed & Pure other & Animal & Other & \\
\hline MDD & $0.7(0.3-1.8)$ & $1.3(0.6-2.8)$ & $2.7(1.4-5.2)$ & $1.0(0.6-1.8)$ & $2.3(1.8-3.9)$ & $1.8(1.1-3.0)$ \\
\hline UP & $0.5(0.2-1.2)$ & $0.7(0.3-1.6)$ & $2.7(1.3-5.6)$ & $0.6(0.3-1.1)$ & $2.0(1.0-3.7)$ & $1.5(0.9-2.8)$ \\
\hline Bipolar disorder & $1.9(0.4-9.1)$ & $9.3(2.1-41.0)$ & $1.2(0.4-3.4)$ & $5.7(1.7-19.0)$ & $3.9(1.2-12.1)$ & $3.6(1.3-9.9)$ \\
\hline Dysthymia & $*$ & $0.7(0.1-5.4)$ & $2.5(0.7-8.4)$ & $0.3(0.0-2.4)$ & $1.9(0.6-5.8)$ & $1.3(0.5-4.1)$ \\
\hline Neurasthenia & $0.8(0.3-2.7)$ & $1.5(0.6-3.6)$ & $2.5(1.0-6.4)$ & $1.1(0.6-2.4)$ & $1.8(1.1-4.8)$ & $1.9(0.9-3.8)$ \\
\hline GAD & $0.8(0.3-2.8)$ & $1.7(0.6-5.0)$ & $1.1(0.5-2.4)$ & $1.3(0.6-2.9)$ & $1.4(0.7-2.6)$ & $1.2(0.7-2.2)$ \\
\hline Agoraphobia & $*$ & $4.9(1.9-13.2)$ & $6.9(2.8-16.8)$ & $2.2(0.9-5.6)$ & $7.7(3.3-18.2)$ & $5.4(2.3-12.5)$ \\
\hline Social phobia & $1.0(0.3-3.7)$ & $1.0(0.3-3.1)$ & $2.2(1.1-4.7)$ & $1.0(0.4-2.4)$ & $1.9(1.0-3.6)$ & $1.6(0.9-3.0)$ \\
\hline OCD & $1.3(0.4-4.8)$ & $4.2(1.6-10.5)$ & $1.3(0.5-3.4)$ & $2.7(1.3-5.8)$ & $2.3(1.1-4.6)$ & $2.1(1.1-4.0)$ \\
\hline Panic disorder & $0.3(0.0-2.3)$ & $1.0(0.3-4.0)$ & $1.5(0.5-4.2)$ & $0.6(0.2-2.0)$ & $1.3(0.5-3.3)$ & $1.0(0.4-2.4)$ \\
\hline Binge eating & $0.7(0.1-5.7)$ & $6.2(1.2-33.2)$ & $4.9(1.4-17.2)$ & $3.3(0.7-14.5)$ & $\mathbf{6 . 6}(2.2-20.2)$ & $5.1(1.7-15.1)$ \\
\hline Daily smoking & $1.5(0.7-3.2)$ & $1.6(0.7-3.5)$ & $0.9(0.6-1.6)$ & $1.6(0.9-2.7)$ & $1.1(0.7-1.8)$ & $1.2(0.8-1.8)$ \\
\hline Alcohol abuse/dependence & $0.8(0.3-2.4)$ & $1.5(0.5-4.5)$ & $1.1(0.4-2.9)$ & $1.1(0.5-2.6)$ & $1.2(0.6-2.7)$ & $1.1(0.6-2.2)$ \\
\hline
\end{tabular}

$M D D$, major depression disorder, $U P$ unipolar depression, $G A D$ generalized anxiety disorder, OCD obsessive-compulsive disorder

* Left blank because of 0 -cells or low frequencies

Significance levels: $p<.05$ (bold)

The other SP represents a heterogeneous group from which the blood-injection-injury (BII) subtype might display more similarities to pure animal phobias than the other subtypes given that disgust is a core feature of both subtypes [13]. Thus, the BII subtype would lend itself as the next candidate for a study comparing pure and mixed SP subtypes. However, since the BII subtype is the smallest subtype, the biasing effect of its misclassification is limited.

This study used data from three major epidemiologic data sets: two of them large and one medium sized. The immediate replication of findings compensates for the uncertainties of data mining. All information was assessed retrospectively and is based on self-reporting, which may constitute a source of bias. Self-reporting of the age of onset of symptoms and other conditions is subject to telescoping effects, which means that the onset took place earlier than reported by the study participants.

The analysis of risk factors was hampered by the different instruments and question formats used in the three studies. Moreover, the studies applied different interview strategies. They took place in different cultural contexts (German and French parts of Switzerland) and involved different generations with different norms and values.

This study included simple association analyses serving for preliminary pattern recognition. Thus, we did not consider correcting for multiple comparisons. The replication in three different databases showed that even analyses of large samples may produce divergent details, the basic patterns remained. More complex and detailed modelling still remains to be done.

\section{Conclusions and outlook}

Conceptualizing subtypes requires introducing both an intensional and an operational definition. Diagnostic manuals and classification systems in psychiatry have paid little attention to the latter. This study on SP illustrates that the choice between alternative operational definitions (in this instance, differentiating between exclusive vs. overlapping subtypes) can make a big difference. Pure animal phobias turned out to be a quite different entity from other SPs. Pure animal phobias and other SPs might be a particularly revealing example, similar to pure and mixed OCD subtypes [34]. Nevertheless, it should be general practice to explicitly justify in any instance not only the intensional but also the operational definition of proposed subtypes.

This study opens the door to several new specific research questions. Above all, the etiopathogenetic mechanisms and risk factors associated with pure animal phobias now appear less clear than ever. Moreover, further research with large data sets needs to show whether the differentiation between pure subtypes in SP is also fruitful in those other than animal phobias.

Last but not least, the findings prompt a series of general questions. First, since pure animal phobias are that different from the mixed SP subtype, research done so far on 
Table 6 Sociodemographic marker variables and risk factors related to specific phobias in the Zurich Study 1979-2008; and calculations based on aggregated 12-month diagnoses from seven interviews

\begin{tabular}{|c|c|c|c|c|c|c|}
\hline & \multicolumn{3}{|c|}{ Exclusive subtypes } & \multicolumn{2}{|c|}{ Overlapping subtypes } & \multirow[t]{2}{*}{ Overall } \\
\hline & Pure animal & Mixed & Pure other & Animal & Other & \\
\hline \multicolumn{7}{|l|}{ Prevalence } \\
\hline$n$ (rate in $\%$, weighted) & $29(5.9)$ & $61(8.3)$ & $54(7.2)$ & $90(14.2)$ & $115(15.5)$ & $144(21.4)$ \\
\hline \multicolumn{7}{|l|}{ Sex } \\
\hline$n$ (rate in $\%$, weighted) males & $5(2.8)$ & $18(3.8)$ & $26(6.9)$ & $23(6.6)$ & $44(10.7)$ & $49(13.5)$ \\
\hline$n$ (rate in $\%$, weighted) females & $24(8.8)$ & $43(12.6)$ & $28(7.6)$ & $67(21.5)$ & $71(20.2)$ & $96(29.1)$ \\
\hline $\begin{array}{l}\text { OR females:males }(95 \% \text { CI) } \\
\text { (weighted) }\end{array}$ & $3.4(1.0-11.7)$ & $3.6(1.4-9.5)$ & $1.1(0.4-2.7)$ & $3.9(1.8-8.5)$ & $2.1(1.1-4.2)$ & $2.6(1.4-4.9)$ \\
\hline Age at onset: mean $(95 \% \mathrm{CI})$ & $9.4(3.9-14.9)$ & $12.1(8.1-16.1)$ & $18.7(15.3-22.1)$ & $11.2(8.1-14.4)$ & $15.2(12.4-18.1)$ & $14.0(11.5-16.6)$ \\
\hline \multicolumn{7}{|l|}{ Education: $n$ (rate in $\%$, weighted) } \\
\hline Low & $19(4.6)$ & $46(10.2)$ & $36(5.5)$ & $65(14.7)$ & $82(15.7)$ & $101(20.3)$ \\
\hline Medium & $7(12.0)$ & $7(4.3)$ & $9(12.5)$ & $14(16.3)$ & $18(16.8)$ & $23(28.9)$ \\
\hline University & $3(5.5)$ & $8(4.3)$ & $9(9.2)$ & $11(9.8)$ & $17(13.5)$ & $21(19.3)$ \\
\hline \multicolumn{7}{|c|}{ Familial aggregation (weighted, ORs $95 \%$ CI) } \\
\hline Parents mentally ill & $1.1(0.2-5.3)$ & $1.3(0.5-3.5)$ & $1.7(0.6-5.0)$ & $1.3(0.5-3.0)$ & $1.5(0.7-3.4)$ & $1.5(0.7-3.1)$ \\
\hline Phobias & 3.0 $(1.0-9.1)$ & $3.3(1.4-8.0)$ & $0.6(0.2-1.9)$ & $3.7(1.8-7.6)$ & $1.8(0.9-3.7)$ & $2.4(1.3-4.5)$ \\
\hline Anxiety & $1.2(0.3-4.0)$ & $\mathbf{3 . 0}(1.2-7.2)$ & $1.8(0.6-4.9)$ & $2.3(1.1-4.8)$ & $2.7(1.3-5.4)$ & $2.3(1.2-4.5)$ \\
\hline Panic & $1.5(0.3-9.8)$ & $2.1(0.5-8.8)$ & $1.2(0.2-8.3)$ & $2.0(0.6-6.7)$ & $1.8(0.5-6.1)$ & $1.9(0.6-5.6)$ \\
\hline Depression & $1.2(0.4-3.7)$ & $1.5(0.6-3.4)$ & $2.6(1.0-6.9)$ & $1.4(0.7-2.8)$ & $2.0(1.0-3.9)$ & $1.9(1.0-3.4)$ \\
\hline Hypomania & $1.0(0.3-3.5)$ & $3.2(1.3-7.7)$ & $2.3(0.8-6.2)$ & $2.2(1.0-4.8)$ & $3.2(1.5-6.5)$ & $2.6(1.3-5.1)$ \\
\hline Alcohol abuse & $1.8(0.4-9.3)$ & $1.5(0.5-4.6)$ & $0.2(0.1-0.6)$ & $1.7(0.7-4.4)$ & $0.8(0.3-2.2)$ & $1.0(0.4-2.5)$ \\
\hline Illicit drugs abuse & $\mathbf{0 . 1}(0.0-1.1)$ & $3.1(0.8-11.5)$ & $0.2(0.1-0.9)$ & $1.9(0.5-7.0)$ & $1.6(0.5-5.6)$ & $1.2(0.4-4.1)$ \\
\hline Smoking & $0.5(0.1-2.0)$ & $1.1(0.4-2.9)$ & $0.4(0.1-1.0)$ & $0.8(0.4-1.9)$ & $0.6(0.3-1.3)$ & $0.6(0.3-1.1)$ \\
\hline \multicolumn{7}{|c|}{ Adversities in family (weighted, ORs $95 \%$ CI) } \\
\hline Run away from home & $*$ & $2.7(0.4-17.6)$ & $0.2(0.0-1.6)$ & $1.7(0.3-10.7)$ & $1.5(0.3-8.8)$ & $1.1(0.2-6.3)$ \\
\hline More severely punished than others & $1.2(0.2-8.1)$ & $4.4(1.6-12.4)$ & $0.2(0.1-0.6)$ & $3.5(1.4-9.0)$ & $2.1(0.9-5.4)$ & $2.0(0.8-4.9)$ \\
\hline Parents did not care enough & $1.1(0.2-6.2)$ & $1.6(0.5-4.8)$ & $1.6(0.5-5.4)$ & $1.5(0.6-3.9)$ & $1.7(0.7-4.2)$ & $1.7(0.7-3.8)$ \\
\hline Quarrels between parents & $1.6(0.4-7.0)$ & $5.4(2.2-13.6)$ & $0.6(0.2-2.2)$ & $4.3(1.9-9.7)$ & $2.8(1.3-6.0)$ & $2.8(1.3-5.7)$ \\
\hline \multicolumn{7}{|l|}{ Other adversity (weighted, ORs $95 \%$ CI) } \\
\hline Afraid of gym or swimming & $2.5(0.6-10.8)$ & $3.3(1.2-8.9)$ & $1.0(0.3-3.7)$ & $3.5(1.4-8.5)$ & $2.3(1.0-5.5)$ & $2.8(1.2-6.3)$ \\
\hline Expelled or unpopular (youth) & $1.2(0.2-7.6)$ & $3.3(1.1-9.5)$ & $1.0(0.2-4.2)$ & $2.7(1.0-7.1)$ & $2.4(0.9-6.0)$ & $2.3(0.9-5.4)$ \\
\hline
\end{tabular}

* Left blank because of 0-cells or low frequencies

Significance levels: $p<.05$ (bold)

animal phobia needs to be re-evaluated. Depending on the proportions of the pure and the mixed subtype, the results might allow for different conclusions. Second, it is not clear which research strategy will be more promising in the future, i.e. whether pure or mixed subtypes are better suited to advance our understanding of the etiopathogenesis of phobias and other mental disorders and, thus, should be investigated first. Third, it is not sure whether the preliminarily established mechanisms are fully transferable, since it cannot be excluded, that the pure and the mixed subtypes imply different etiopathogenetic mechanisms and also partly different neurobiological backgrounds. Fourth, the proximity of pure subtypes and unimorbid diagnoses suggests that it might be interesting to raise all these questions with respect to unimorbid diagnoses, i.e. beyond SP and OCD. 
Table 7 Comorbidity patterns in specific phobias in the Zurich Study 1979-2008; odds ratios and confidence intervals (95\%); and calculations based on aggregated 12-month diagnoses from seven interviews

\begin{tabular}{|c|c|c|c|c|c|c|}
\hline & \multicolumn{3}{|c|}{ Exclusive subtypes } & \multicolumn{2}{|c|}{ Overlapping subtypes } & \multirow[t]{2}{*}{ Overall } \\
\hline & Pure animal & Mixed & Pure other & Animal & Other & \\
\hline UP & $2.4(0.7-7.9)$ & $1.7(0.6-5.0)$ & $0.8(0.2-2.7)$ & $2.1(0.9-5.0)$ & $1.3(0.5-3.0)$ & $1.7(0.8-3.6)$ \\
\hline Bipolar disorder & $1.8(0.3-11.4)$ & $5.3(1.6-17.6)$ & $3.0(0.7-12.5)$ & $4.4(1.5-13.2)$ & $5.6(1.9-16.3)$ & $5.7(2.0-16.1)$ \\
\hline Dysthymia & $0.5(0.1-2.5)$ & $12.8(4.3-37.7)$ & $0.7(0.2-2.2)$ & $8.4(3.0-23.2)$ & $7.3(2.8-19.1)$ & $5.9(2.3-15.0)$ \\
\hline Neurasthenia & $2.0(0.4-9.4)$ & $3.0(0.9-10.2)$ & $0.4(0.1-1.2)$ & $2.8(1.0-8.0)$ & $1.7(0.6-4.9)$ & $1.9(0.7-5.0)$ \\
\hline GAD & $1.6(0.5-5.2)$ & $3.1(1.3-7.6)$ & $2.7(1.0-7.0)$ & $2.6(1.2-5.5)$ & $3.3(1.6-6.7)$ & $3.1(1.6-5.9)$ \\
\hline Agoraphobia & $1.1(0.3-4.5)$ & $11.1(4.5-27.4)$ & $3.7(1.4-9.6)$ & $5.7(2.7-12.0)$ & $9.2(4.5-19.1)$ & $6.9(3.5-13.6)$ \\
\hline Social phobia & $1.1(0.2-5.3)$ & $8.4(3.3-21.4)$ & $1.7(0.6-4.9)$ & $\mathbf{5 . 0}(2.2-11.7)$ & $5.6(2.5-12.5)$ & $4.5(2.1-9.9)$ \\
\hline OCD & $2.6(0.6-10.8)$ & $3.4(1.1-10.4)$ & $1.3(0.3-5.3)$ & $3.5(1.3-9.1)$ & $2.5(1.0-6.6)$ & $\mathbf{3 . 0}(1.3-7.3)$ \\
\hline Panic disorder & $0.5(0.1-1.7)$ & $6.4(2.0-20.4)$ & $1.2(0.5-3.0)$ & $3.7(1.2-10.8)$ & $4.2(1.5-11.6)$ & $3.1(1.2-8.4)$ \\
\hline Binge eating & $2.3(0.4-13.4)$ & $3.0(0.9-9.8)$ & $1.0(0.2-6.5)$ & $3.1(1.1-9.0)$ & $2.2(0.8-6.3)$ & $2.5(0.9-6.8)$ \\
\hline Marijuana abuse & $0.2(0.0-0.6)$ & $2.4(0.8-7.1)$ & $0.6(0.1-3.8)$ & $1.3(0.5-3.5)$ & $1.5(0.6-3.9)$ & $1.0(0.4-2.6)$ \\
\hline Alcohol abuse & $1.8(0.6-5.7)$ & $1.2(0.4-3.0)$ & $1.3(0.5-3.5)$ & $1.5(0.7-3.1)$ & $1.2(0.6-2.6)$ & $1.5(0.8-2.8)$ \\
\hline Illicit drugs abuse & $\mathbf{0 . 2}(0.1-0.8)$ & $3.0(0.9-9.5)$ & $1.0(0.2-5.0)$ & $1.6(0.6-4.8)$ & $2.1(0.8-5.7)$ & $1.5(0.6-3.8)$ \\
\hline
\end{tabular}

$U P$ unipolar depression, $G A D$ generalized anxiety disorder, $O C D$ obsessive-compulsive disorder

Significance levels: $p<.05$ (bold)

Acknowledgments The Zurich Study was supported by the Swiss National Science Foundation (most recent Grant Number 32-50881.97). ZInEP was supported by a private donation. The donor had no further role in the experimental design, the collection, analysis, and interpretation of data, the writing of this report or the decision to submit this paper for publication. The ZInEP Epidemiology Survey, in particular research in the Centre for Neuro- and Sociophysiology, was supported by the Swiss National Science Foundation (Grant \#3247B0-122071). The PsyCoLaus study was supported by research grants from GlaxoSmithKline, the Faculty of Biology and Medicine of Lausanne, and the Swiss National Science Foundation (Grants 3200B0-105993, 3200B0-118308, 33CSCO-122661, 33CS30-139468 and 33CS30-148401)

\section{Compliance with ethical standards}

Conflict of interest The authors declare that they have no conflict of interest.

\section{References}

1. Ajdacic-Gross V, Horvath S, Canjuga M, Gamma A, Angst J, Rossler W, Eich D (2006) How ubiquitous are physical and psychological complaints in young and middle adulthood? A longitudinal perspective. Soc Psychiatry Psychiatr Epidemiol 41:881-888

2. Ajdacic-Gross V, Muller M, Rodgers S, Warnke I, Hengartner MP, Landolt K, Hagenmuller F, Meier M, Tse LT, Aleksandrowicz A, Passardi M, Knopfli D, Schonfelder H, Eisele J, Rusch N, Haker H, Kawohl W, Rossler W (2014) The ZInEP epidemiology survey: background, design and methods. Int J Methods Psychiatr Res 23:451-468

3. Angst J, Azorin JM, Bowden CL, Perugi G, Vieta E, Gamma A, Young AH (2011) Prevalence and characteristics of undiagnosed bipolar disorders in patients with a major depressive episode: the bridge study. Arch Gen Psychiatry 68:791-798
4. Angst J, Dobler-Mikola A, Binder J (1984) The Zurich studya prospective epidemiological study of depressive, neurotic and psychosomatic syndromes. I. Problem, methodology. Eur Arch Psychiatry Neurol Sci 234:13-20

5. Angst J, Gamma A, Neuenschwander M, Ajdacic-Gross V, Eich D, Rössler W, Merikangas KR (2005) Prevalence of mental disorders in the Zurich cohort study: a twenty year prospective study. Epidemiologia e Psichiatria Sociale 14:68-76

6. Becker ES, Rinck M, Turke V, Kause P, Goodwin R, Neumer S, Margraf J (2007) Epidemiology of specific phobia subtypes: findings from the dresden mental health study. Eur Psychiatry 22:69-74

7. Benjet C, Borges G, Stein DJ, Mendez E, Medina-Mora ME (2012) Epidemiology of fears and specific phobia in adolescence: results from the mexican adolescent mental health survey. J Clin Psychiatry 73:152-158

8. Berney A, Preisig M, Matthey ML, Ferrero F, Fenton BT (2002) Diagnostic interview for genetic studies (DIGS): inter-rater and test-retest reliability of alcohol and drug diagnoses. Drug Alcohol Depend 65:149-158

9. Bernstein DP, Fink L, Handelsman L, Foote J, Lovejoy M, Wenzel K, Sapareto E, Ruggiero J (1994) Initial reliability and validity of a new retrospective measure of child abuse and neglect. Am J Psychiatry 151:1132-1136

10. Bianchi KN, Carter MM (2012) An experimental analysis of disgust sensitivity and fear of contagion in spider and blood injection injury phobia. J Anxiety Disord 26:753-761

11. Burstein M, Georgiades K, He JP, Schmitz A, Feig E, Khazanov GK, Merikangas K (2012) Specific phobia among U.S. adolescents: phenomenology and typology. Depress Anxiety 29:1072-1082

12. Choy Y, Fyer AJ, Goodwin RD (2007) Specific phobia and comorbid depression: a closer look at the national comorbidity survey data. Compr Psychiatry 48:132-136

13. Cisler JM, Olatunji BO, Lohr JM (2009) Disgust, fear, and the anxiety disorders: a critical review. Clin Psychol Rev 29:34-46

14. Cuthbert BN, Insel TR (2013) Toward the future of psychiatric diagnosis: the seven pillars of rdoc. BMC Med 11:126 
15. Czajkowski N, Kendler KS, Tambs K, Roysamb E, ReichbornKjennerud T (2011) The structure of genetic and environmental risk factors for phobias in women. Psychol Med 41:1987-1995

16. Del Casale A, Ferracuti S, Rapinesi C, Serata D, Piccirilli M, Savoja V, Kotzalidis GD, Manfredi G, Angeletti G, Tatarelli R, Girardi P (2012) Functional neuroimaging in specific phobia. Psychiatry Res 202:181-197

17. Derogatis LR, Cleary PA (1977) Confirmation of the dimensional structure of the SCL-90-R: a study in construct validation. J Clin Psychol 33:981-989

18. Dunn G, Pickles A, Tansella M, Vazquez-Barquero JL (1999) Two-phase epidemiological surveys in psychiatric research. Br J Psychiatry 174:95-100

19. Endicott J, Spitzer RL (1978) A diagnostic interview: the schedule for affective disorders and schizophrenia. Arch Gen Psychiatry $35: 837-844$

20. Firmann M, Mayor V, Vidal PM, Bochud M, Pecoud A, Hayoz D, Paccaud F, Preisig M, Song KS, Yuan X, Danoff TM, Stirnadel HA, Waterworth D, Mooser V, Waeber G, Vollenweider P (2008) The CoLaus study: a population-based study to investigate the epidemiology and genetic determinants of cardiovascular risk factors and metabolic syndrome. BMC Cardiovasc Disord 8:6

21. Hardt J, Egle UT, Kappis B, Hessel A, Brahler E (2004) Symptom checklist SCL-27. Psychother Psychosom Med Psychol 54:214-223

22. Hochstrasser B, Angst J (1996) The Zurich Study: XXII. Epidemiology of gastrointestinal complaints and comorbidity with anxiety and depression. Eur Arch Psychiatry Clin Neurosci 246:261-272

23. Kessler RC, Ormel J, Petukhova M, McLaughlin KA, Green JG, Russo LJ, Stein DJ, Zaslavsky AM, Aguilar-Gaxiola S, Alonso J, Andrade L, Benjet C, de Girolamo G, de Graaf R, Demyttenaere K, Fayyad J, Haro JM, Hu C, Karam A, Lee S, Lepine JP, Matchsinger H, Mihaescu-Pintia C, Posada-Villa J, Sagar R, Ustun TB (2011) Development of lifetime comorbidity in the world health organization world mental health surveys. Arch Gen Psychiatry 68:90-100

24. Leboyer M, Maier W, Teherani M, Lichtermann D, D'Amato T, Franke P, Lepine JP, Minges J, McGuffin P (1991) The reliability of the SADS-LA in a family study setting. Eur Arch Psychiatry Clin Neurosci 241:165-169

25. Lueken U, Kruschwitz JD, Muehlhan M, Siegert J, Hoyer J, Wittchen HU (2011) How specific is specific phobia? Different neural response patterns in two subtypes of specific phobia. Neuroimage 56:363-372

26. Merikangas KR, Zhang H, Avenevoli S, Acharyya S, Neuenschwander M, Angst J (2003) Longitudinal trajectories of depression and anxiety in a prospective community study: THE zurich cohort study. Arch Gen Psychiatry 60:993-1000

27. Novy J, Castelao E, Preisig M, Vidal PM, Waeber G, Vollenweider P, Rossetti AO (2012) Psychiatric co-morbidities and cardiovascular risk factors in people with lifetime history of epilepsy of an urban community. Clin Neurol Neurosurg 114:26-30

28. Nurnberger JI Jr, Blehar MC, Kaufmann CA, York-Cooler C, Simpson SG, Harkavy-Friedman J, Severe JB, Malaspina D, Reich T (1994) Diagnostic interview for genetic studies.
Rationale, unique features, and training. NIMH genetics initiative. Arch Gen Psychiatry 51:849-859

29. Ollendick TH, Raishevich N, Davis TE 3rd, Sirbu C, Ost LG (2010) Specific phobia in youth: phenomenology and psychological characteristics. Behav Ther 41:133-141

30. Oosterink FM, de Jongh A, Hoogstraten J (2009) Prevalence of dental fear and phobia relative to other fear and phobia subtypes. Eur J Oral Sci 117:135-143

31. Park S, Sohn JH, Hong JP, Chang SM, Lee YM, Jeon HJ, Cho SJ, Bae JN, Lee JY, Son JW, Cho MJ (2013) Prevalence, correlates, and comorbidities of four DSM-IV specific phobia subtypes: results from the korean epidemiological catchment area study. Psychiatry Res 209:596-603

32. Preisig M, Fenton BT, Matthey ML, Berney A, Ferrero F (1999) Diagnostic interview for genetic studies (digs): inter-rater and test-retest reliability of the french version. Eur Arch Psychiatry Clin Neurosci 249:174-179

33. Preisig M, Waeber G, Vollenweider P, Bovet P, Rothen S, Vandeleur C, Guex P, Middleton L, Waterworth D, Mooser V, Tozzi F, Muglia P (2009) The PsyCoLaus study: methodology and characteristics of the sample of a population-based survey on psychiatric disorders and their association with genetic and cardiovascular risk factors. BMC Psychiatry 9:9

34. Rodgers S, Ajdacic-Gross V, Kawohl W, Muller M, Rossler W, Hengartner MP, Castelao E, Vandeleur C, Angst J, Preisig M (2015) Comparing two basic subtypes in OCD across three large community samples: a pure compulsive versus a mixed obsessive-compulsive subtype. Eur Arch Psychiatry Clin Neurosci 265:719-734

35. Trumpf J, Margraf J, Vriends N, Meyer AH, Becker ES (2010) Predictors of specific phobia in young women: a prospective community study. J Anxiety Disord 24:87-93

36. van Duinen MA, Schruers KR, Griez EJ (2010) Desynchrony of fear in phobic exposure. J Psychopharmacol 24:695-699

37. Watson D, Clark LA (2006) Clinical diagnosis at the crossroads. Clin Psychol Sci Pract 13:210-215

38. Wittchen HU, Jacobi F, Rehm J, Gustavsson A, Svensson M, Jonsson B, Olesen J, Allgulander C, Alonso J, Faravelli C, Fratiglioni L, Jennum P, Lieb R, Maercker A, van Os J, Preisig M, Salvador-Carulla L, Simon R, Steinhausen HC (2011) The size and burden of mental disorders and other disorders of the brain in Europe 2010. Eur Neuropsychopharmacol 21:655-679

39. Woody SR, McLean C, Klassen T (2005) Disgust as a motivator of avoidance of spiders. J Anxiety Disord 19:461-475 